\title{
Evaluation of a topical treatment for the relief of sensitive skin
}

\author{
Ingrid R Heinicke' \\ Damian H Adams ${ }^{2}$ \\ Tanya M Barnes' \\ Kerryn A Greive'
}

'Ego Pharmaceuticals, Braeside, VIC, Australia; ${ }^{2}$ Mawson Institute, University of South Australia, Mawson Lakes, SA, Australia

Correspondence: Kerryn A Greive Ego Pharmaceuticals Pty Ltd, 2I-3I Malcolm Road, Braeside, VIC 3195, Australia

Tel 6 I 395868874

Fax 6I 395807647

Email kerryng@egopharm.com
This article was published in the following Dove Press journal:

Clinical, Cosmetic and Investigational Dermatology

27 July 2015

Number of times this article has been viewed

Background: Approximately, 50\% of the population claim to have sensitive skin, which has created an important challenge for dermatologists and the cosmetic industry. This study evaluates the properties of QV Face Rescue Gel (Rescue Gel) that contains a combination of moisturizing and anti-irritant ingredients, and which is used to relieve the symptoms of sensitive facial skin.

Methods: The ability of Rescue Gel to induce collagen types I and III in cultured neonatal human foreskin fibroblasts compared to transforming growth factor beta 1, a known potent inducer of collagen types I and III, was measured using immunofluorescence staining. Furthermore, healthy volunteers were recruited to measure the potential for Rescue Gel to reduce erythema induced by solar-simulated ultraviolet radiation on the skin compared to $0.5 \%$ hydrocortisone cream (positive control) as well as it's ability to decrease transepidermal water loss compared to baseline levels. In addition, the formulation was tested for its potential to be 1) nonstinging using a facial sting/discomfort assay performed on volunteers who reacted positively to lactic acid, 2) nonirritating as determined by repeat insult patch tests, and 3) noncomedogenic.

Results: Rescue Gel significantly induced collagen types I and III in cultured human foreskin fibroblasts similarly to transforming growth factor beta 1. In volunteers, Rescue Gel was shown to significantly reduce erythema induced by solar-simulated ultraviolet radiation similarly to $0.5 \%$ hydrocortisone, and to significantly reduce transepidermal water loss compared to baseline levels. Further, the formulation was found to be nonstinging, nonirritating, and noncomedogenic. No adverse events were observed.

Conclusion: In this study, Rescue Gel has been shown to exhibit properties that make it effective for use on sensitive or irritated facial skin, without exacerbation of the symptoms associated with sensitive skin.

Keywords: sensitive skin, inflammation, moisturization, collagen, erythema

\section{Introduction}

Sensitive skin is an increasingly common condition with approximately $50 \%$ of women and $40 \%$ of men having self-diagnosed sensitive skin. ${ }^{1,2}$ Sensitive skin is characterized by subjective complaints of discomfort without visible signs of irritation. Sensory symptoms of sensitive skin include stinging, burning, tightness, and sensation of pain. Dryness or impaired hydration of skin may also be present. Erythema is sometimes, but not necessarily, associated with sensitive skin. Sensitive skin may be triggered by hypersensitivity to a range of stimuli which may be physical, chemical, psychological, or hormonal. ${ }^{2,3}$

The pathophysiology of sensitive skin is poorly understood and is complex, since multiple factors such as age, skin pigmentation, anatomic region, cultural factors, 
environmental factors, and preexisting diseases have influence. ${ }^{2,3}$ Studies suggest that sensitive skin is the result of impaired barrier function of the stratum corneum, which leads to the exposure of immune system cells and sensitive nerves, resulting in marked cutaneous responses to otherwise harmless stimuli. ${ }^{2,3}$ Sensitive skin is most common on the face, but can be found on other areas such as the hands or scalp. ${ }^{1}$

The increased prevalence of sensitive skin has created a challenge for dermatologists and the cosmetic industry with a sizeable demand for products designed to minimize skin sensitivity. ${ }^{2,4}$ Ingredients in products designed for sensitive skin should be carefully selected to minimize exacerbation of symptoms. Products should be free from sensitizers and common irritants such as fragrance, herbal or essential oil extracts, propylene glycol, and lanolin. Products should include ingredients that moisturize sensitive skin, as this is important to decrease transepidermal water loss (TEWL) and repair barrier function. Anti-inflammatory ingredients should also be included to reduce redness and stinging, which in turn increases consumer product tolerance. ${ }^{4}$ Ingredients that induce collagen would be beneficial to maintain strength and durability for healthy skin. ${ }^{5}$

To date, there have been few published studies on the effectiveness of topical products to relieve the symptoms of sensitive skin, with most studies concentrating on individual ingredients. The purpose of this study was to evaluate the properties of a product that contains a combination of moisturizing and anti-irritant ingredients, which is used to relieve the sensations of sensitive skin on the face. Specifically, the ability of QV Face Rescue Gel (Rescue Gel; Ego Pharmaceuticals Pty Ltd, Braeside, VIC, Australia) to induce collagen types I and III in cultured neonatal human foreskin fibroblasts (HFFs) was measured using immunofluorescence staining. Healthy volunteers were recruited to measure the potential of Rescue Gel to reduce erythema induced by solar-simulated ultraviolet (UV) radiation and to decrease TEWL. In addition, the formulation was tested for its potential to be nonstinging, nonirritating, and noncomedogenic.

\section{Materials and methods Topical preparations}

Rescue Gel contains glycerin, paraffinum liquidum, Hamamelis virginiana (witch hazel) water, niacinamide, panthenol, disodium lauriminodipropionate tocopherol phosphate (DLTP), and Avena sativa (oat) kernel extract (Ego Pharmaceuticals Pty Ltd). Acetulan contains cetyl acetate and acetylated lanolin alcohol (The Lubrizol Corporation, Wickliffe, OH, USA). DermAid Soft 0.5\% AUST R 71683 cream contains $0.5 \%$ hydrocortisone (Ego Pharmaceuticals Pty Ltd).

\section{Cell culture and collagen regulation}

Neonatal HFFs donated by Dr Pritinder Kaur (Epithelial Stem Cell Biology Laboratory, Peter MacCallum Cancer Centre, Melbourne, VIC, Australia) were cultured in Dulbecco's Modified Eagles Medium (DMEM) with 10\% fetal bovine serum and penicillin-streptomycin at $37^{\circ} \mathrm{C}$ and $5 \% \mathrm{CO}_{2}$. At confluence, HFFs were trypsinized and seeded into 96 -well plates at $5 \times 10^{5}$ cells $/ \mathrm{mL}(100 \mu \mathrm{L} ; 50,000$ cells $)$ and cultured for 24 hours. Media was removed and replaced with serum-free DMEM containing penicillin-streptomycin for 6 hours prior to exposure to Rescue Gel diluted 1:200 in serum-free DMEM containing penicillin-streptomycin and then incubated at $37^{\circ} \mathrm{C}$ and $5 \% \mathrm{CO}_{2}$ for 60 hours. The dilution of 1:200 was determined to be optimal through analysis of cellular behavior and collagen expression via test trial exposure of HFFs to Rescue Gel (data not shown). The positive control was transforming growth factor beta 1 (TGF- $\beta 1$ ), a known inducer of collagen types $\mathrm{I}^{6}$ and III, ${ }^{7}$ diluted to $5 \mathrm{ng} / \mathrm{mL}$ in serum-free DMEM with penicillinstreptomycin. The negative control was serum-free DMEM with penicillin-streptomycin and the blank consisted of media without cells.

After 60 hours, cells were fixed and permeabilized in ice cold methanol for 15 minutes at $-20^{\circ} \mathrm{C}$. Immunofluorescence staining was performed using anticollagen type I or III as primary antibody (Rockland Immunochemicals Inc., Philadelphia, PA, USA) and Alexa Fluor 488 goat anti-rabbit IgG as secondary antibody (Life Technologies, Carlsbad, CA, USA). Plates/cells were photographed utilizing cellSens Dimension software (Olympus Corporation, Tokyo, Japan) with an Olympus DP80 camera utilizing a $1.45 \mathrm{MP}, 14$ bit, monochrome CCD sensor on an Olympus IX83 fluorescence microscope running CoolLED pE light source with $490 \mathrm{~nm}$ LED and a Märzhäuser Wetzlar Tango Desktop motorized stage controller. Photographic images of cells (40× magnification) were automatically stitched together and gray scale intensity was calculated using the cellSens Dimension software.

\section{Erythema reduction study}

All human studies were conducted in accordance with the National Health and Medical Research Council (NH\&MRC) of Australia guidelines and ICH Guidelines for Good Clinical Practice, and had approval from institutional boards (AMA Laboratories, Inc., New City, NY, USA; Cantor Research 
Laboratories, Inc., Blauvelt, NY, USA; Dermatest Pty Ltd, Rockdale, NSW, Australia). Seven human volunteers aged 18-65 years were selected with pale, minimally sun exposed back skin, free of confounding blemishes. The light source used was a $150 \mathrm{~W}, 16 \mathrm{~S}$ single port solar simulator (Solar Light Co, Philadelphia, PA, USA). The methodology for this study was derived from Ibbotson et al. ${ }^{8}$ On day 1 , volunteer's minimal erythemal dose (MED) on unprotected skin (control site) on the back was determined following a series of UV exposures with 1-second increments between each exposure. The volunteer's MED was determined to be the time per dose of exposure that produced the minimal perceptible erythema, observed 16-24 hours postexposure. Skin erythema was evaluated by visual observation under constant tungsten illumination, and under conditions where room lighting was designed for red color rendering. The color acuity of the operators was evaluated by the Farnsworth Munsel Test and the US Color Test, and found to be in the top $1 \%$.

On day 2, one test site for each test product $\left(30 \mathrm{~cm}^{2}\right)$ was delineated and outlined. Each test site was divided into five subsite areas that were at least $1 \mathrm{~cm}^{2}$ with $>1 \mathrm{~cm}$ between subsites. Volunteers received a series of UV exposures with 1 -second increments; three below the MED, one at the MED, and one above the MED. Following the exposures, either no treatment, $0.5 \%$ hydrocortisone cream (positive control), or Rescue Gel were applied on skin at a dose of $4 \mathrm{mg} / \mathrm{cm}^{2}$ and rubbed in well in two applications, one immediately following the exposures and one 4-5 hours later in a double-blind manner. Response subsites were scored by visual comparison on day 3, 16-24 hours postexposure. The value of 1.0 was accorded to the MED. Responses of greater intensities were accorded values of 1.25-1.75, lesser intensities $0.25-0.75$, and no response 0 . All test sites were photographed with a digital camera. Response subsites were scored again on day 4 and 5 , and the average scores for days 3-5 determined for each test product.

\section{TEWL study}

Inclusion criteria were: males and females aged 18 to 70 years, not taking medication or under the care of a doctor for a period of 1 month prior to commencement and throughout the entire test period, completed an extensive medical history form, free of any dermatologica1 or systemic disorder that would interfere with the results, available for the study duration, and gave written informed consent. Exclusion criteria were: individuals under a doctor's care, taking medication that could mask or interfere with test results, known hypersensitivity to cosmetic products, any form of skin cancer or any disease that could interfere with test results, diagnosed with chronic skin allergies, excessive hair on the test sites, and females who indicated that they were pregnant or nursing an infant.

Ten healthy human volunteers aged 30-54 years (one male and nine female) were enrolled in the study. In order to precondition the test sites and to keep topical treatments constant for all test subjects, volunteers were required to abstain from using deodorant soaps, moisturizing soaps, or cosmetic moisturizers on the test area for a period of 7 days prior to study commencement and during the course of the study. At the completion of the washout period, volunteers returned to the test facility where a single application of Rescue Gel was liberally applied through plastic volumetric syringes and evenly spread using a glass rod to a rectangular area of the inner forearm measuring $2.5 \times 10 \mathrm{~cm}^{2}$. Volunteers were blinded as to the nature of the material being applied.

Measurement of TEWL was performed using a TEWA Meter (Model TM 210 TEWA Meter; Courage and Khazaka Electronic GmbH, Cologne, Germany). This instrument measures the water barrier function of the skin via a probe that indirectly measures the density gradient of water evaporation from the skin by two pairs of sensors, temperature and relative humidity, and is expressed in $\mathrm{g} / \mathrm{hm}^{2}$. Even the slightest damage to the skin water barrier can be determined at an early stage, where a higher value indicates greater damage to the stratum corneum. Before each set of measurements, volunteers were required to equilibrate in a closed environment with a constant temperature $\left(20^{\circ} \mathrm{C} \pm 2^{\circ} \mathrm{C}\right)$ and humidity (45\%-55\% RH [relative humidity]). Measurements were taken in a double-blind manner at $t=0$ (preapplication) and at 2, 4 and 7 hours post-application at five different points on the skin, giving a total of 50 points for each time point. Volunteers were required to remain in the laboratory for the duration of the study.

The percentage change in TEWL was determined by the following equation: Percentage change $=[(a-b) / b] \times 100$, where $a=$ individual value of TEWL at each individual time point and $b=$ zero hour value of TEWL.

\section{Facial sting/discomfort study}

This study was adapted from Frosch and Kligman and performed in a double-blind manner. ${ }^{9}$ Ten healthy female human volunteers aged 31-68 years were enrolled in the study according to the inclusion/exclusion criteria outlined for the TEWL study, as well as being lactic acid sensitive. Volunteers were prescreened for their sensitivity to topically applied materials on day 1 of the study. Upon arrival at the laboratory, volunteers washed their face with a Dove 
Beauty Bar (Unilever Australasia, Epping, NSW, Australia). One milliliter of $10 \%$ lactic acid or $1 \mathrm{~mL}$ of distilled water on cotton swabs was randomly and concurrently applied to either side of the face of volunteers. Volunteers were instructed to record any sensations perceived as discomfort immediately after application and again 2.5 and 5 minutes post-application using the scale: $0=$ none; $0.5=$ barely perceptible; $1.0=$ slightly perceptible; $1.5=$ definitely perceptible; $2.0=$ moderately perceptible; $2.5=$ dramatically perceptible; $3.0=$ severely perceptible.

To determine whether a volunteer was lactic acid sensitive, their cumulative score taken at 2.5 and 5 minutes post-application was used, calculated for each side of the face according to the following scale: nonsensitive: no reaction to lactic acid or $<1.0$; sensitive: 1.0-2.0; extra/ super sensitive: $>2.0$. To assess the effect of Rescue Gel on sensitive skin, only volunteers exhibiting cumulative scores 1.0-2.0 for lactic acid were inducted into the study.

Rescue Gel or a distilled water control was randomly assigned to alternating sides of the face and applied by a technician in a double-blind manner. One milliliter of test product on a cotton swab was liberally spread in a smooth motion starting from the nasolabial fold and across the upper cheek area. Volunteers were seated in an environmental chamber at $95-100^{\circ} \mathrm{F}$ and $75 \%-80 \% \mathrm{RH}$. All the sensations felt at $0,2.5$, and 5 minutes were recorded with burning, drying, stinging, smarting, tingling, prickly, itching, and warm/ hot considered to be relevant responses. The intensity of the sensation was recorded as described earlier.

A test product was deemed nonstinging if it exhibited a delayed mean sting score of 0.5 or less and/or if it exhibited a delayed mean sting score equivalent to the reference control, regardless of the magnitude of the delayed mean sting score.

\section{Irritation and sensitization potential study}

One hundred and seven healthy human volunteers aged 19-67 years (28 male and 79 female) were enrolled in the study according to the inclusion/exclusion criteria outlined for the TEWL study. Volunteers were requested to bathe or wash as usual before the test. Rescue Gel, $0.2 \mathrm{~mL}$, was placed onto a semiocclusive, hypoallergenic patch (Parke-Davis Hypoallergenic Readi Bandage; $20 \times 20 \mathrm{~mm}^{2}$ Webril affixed to the center of a $40 \times 40 \mathrm{~mm}$ adhesive bandage). The patch was then affixed directly to the skin of the infrascapular regions of the back, to the right or left of the midline and the volunteer was allowed to return home with instructions not to wet or expose the test area to direct sunlight.
After 24 hours, the patch was removed by the participant at home. This procedure was repeated until a series of nine consecutive 24-hour exposures were made for every Monday, Wednesday, and Friday for 3 consecutive weeks.

In the event of an adverse reaction, the area of erythema and edema was measured. Edema was estimated by the evaluation of the skin with respect to the contour of the unaffected normal skin. Reactions were scored just before applications two through nine and the next test date following application nine. Participants were then given a 10-14-day rest period after which a challenge or retest dose was applied once to a previously unexposed test site. The retest dose was equivalent to any one of the original nine exposures. Reactions were scored 24 and 48 hours after application.

\section{Comedogenic potential study}

The methodology for this study was based on the study of Mills and Kligman. ${ }^{10}$ Six healthy female human volunteers aged 21-36 years, prescreened for prominent follicular orifices on the medial region of the upper back, were included in the study. If necessary, follicular biopsy technique was used to further qualify volunteers for inclusion according to microscopic results. Thrice weekly, 0.2-0.5 mL Rescue Gel or the positive control Acetulan was dispensed onto a $4 \times 4 \mathrm{~cm}^{2}$ cotton cloth patch and applied to the medial region of the back and secured to the skin by semiocclusive hypoallergenic tape, using an overlayer of adhesive taping in a double-blind manner. An untreated negative control site was included. This procedure was repeated until three applications per week were accomplished for a total of 6 weeks. On removal, all sites were evaluated for any overt signs of irritation prior to repatching.

A series of follicular biopsies were performed at induction and following the final patch removal. A total of five, $1 \mathrm{~cm}^{2}$ area biopsies were taken, randomly selected from the $16 \mathrm{~cm}^{2}$ grid comprising each test site. Slides were examined under a microscope and the numbers of follicles and microcomedones per square centimeter were counted. An optical micrometer (Olympus Life Science Solutions, Tokyo, Japan) was employed to measure the size of several microcomedones and follicles to provide a reference. The mean numbers of follicles and microcomedones were then calculated for each test site. Upon completion of counting, comedogene scoring was based on the following scale: $0=$ no microcomedones or hyperkeratinization and normal follicular orifices; $1=$ at least $25 \%$ of the follicles exhibiting microcomedones (small keratinaceous cylinders inspissated within the lumina); $2=$ at least $50 \%$ of the follicles exhibiting moderately sized 
microcomedones; $3=$ at least $75 \%$ of the follicles displaying large, tightly compacted, globoid microcomedones.

Data were expressed as a mean follicle:mean comedone ratio. To determine the change in the comedone activity due to the test agent, a numeric value for the ratio was obtained (Table 1). For example, for Acetulan pretreatment $19.85: 1=1 / 19.85=0.0504$, and post-treatment $12.74: 1=1 / 12.74=0.0785$. The values obtained were used to determine the percentage difference in comedone activity due to treatment. For example, for Acetulan, the percentage difference in activity was $(0.0785-0.0504) / 0.0504 \times 100=55.8 \%$.

\section{Statistical analysis}

The Student's $t$-test was used for statistical analysis where $P<0.05$ was considered statistically significant. Results are expressed as mean \pm standard error of mean (SEM).

\section{Results}

\section{Collagen regulation}

Immunofluorescence staining for intracellular collagen type I showed a significant increase in staining following treatment of cells with Rescue Gel diluted 1:200 compared to untreated cells $(P=0.0007$; Figure 1$)$ and was similar to that obtained for the positive control TGF- $\beta 1(5 \mathrm{ng} / \mathrm{mL})$, a known potent inducer of collagen type I. ${ }^{6}$

Similar results were also obtained with intracellular collagen type III immunofluorescence staining, with a significant increase in staining following treatment of cells with Rescue Gel diluted 1:200 compared to untreated cells $(P<0.0001$; Figure 2) and was similar to that obtained for TGF- $\beta 1$ $(5 \mathrm{ng} / \mathrm{mL})$, a known potent inducer of collagen type III.?

\section{Erythema reduction study}

All the seven volunteers completed the study. Figure 3 illustrates the mean total erythema scores evaluated and scored by visual comparison following a series of UV-simulated irradiations. A significant decrease in erythema was observed following treatment with both the positive control $0.5 \%$ hydrocortisone $(4.1 \pm 1.0, \mathrm{n}=7, P=0.01)$ and Rescue Gel (4.1 $\pm 0.9, \mathrm{n}=7, P=0.036$,), compared to no treatment $(6.5 \pm 1.6$, $\mathrm{n}=7)$. No adverse events were reported.

\section{TEWL study}

All ten volunteers completed the study with no adverse reactions observed. The preapplication mean TEWL value was $4.57 \pm 0.63 \mathrm{~g} / \mathrm{hm}^{2}$. Figure 4 illustrates the mean percentage decrease in TEWL over time compared to baseline. Two hours post-application, TEWL significantly decreased by approximately $37 \%$ ( $P=0.026$ vs $t=0)$, and by $49 \%$ at both $t=4$ hours $(P=0.006$ vs $t=0)$ and $t=7$ hours $(P=0.002$ vs $t=0)$ post-application compared to baseline. These results show that a significant reduction in water loss was observed 2 hours following the topical application of Rescue Gel, which continued to improve for the duration of the study.

\section{Facial sting/discomfort study}

All ten volunteers reacted positively to lactic acid and were thus enrolled and completed the study. Only two of the ten volunteers noted any facial discomfort for Rescue Gel that was rated as being "barely perceptible" according to the discomfort/sting intensity scale. No adverse reactions were observed. The mean facial sting score for Rescue Gel was $0.075 \pm 0.053, \mathrm{n}=10$, compared with 0 for distilled water. Therefore, Rescue Gel was considered to be nonstinging.

\section{Irritation and sensitization potential study}

One hundred volunteers completed the study with no adverse reactions of any kind observed during the course of the study. Therefore, Rescue Gel was considered to be nonirritating and nonsensitizing to the skin.

\section{Comedogenic potential study}

All six volunteers completed the study and no adverse reactions were observed. Table 1 shows a clinically significant

Table I Mean number of follicles $/ \mathrm{cm}^{2}$, mean number of comedones $/ \mathrm{cm}^{2}$, and calculation of comedone score

\begin{tabular}{|c|c|c|c|c|c|c|c|c|c|c|}
\hline \multirow[t]{2}{*}{ Test product } & \multicolumn{2}{|c|}{ Mean follicles $/ \mathrm{cm}^{2}$} & \multicolumn{2}{|c|}{ Mean comedones $/ \mathrm{cm}^{2}$} & \multicolumn{2}{|c|}{$\begin{array}{l}\text { Follicles: } \\
\text { comedones }\end{array}$} & \multicolumn{2}{|c|}{$\begin{array}{l}\text { \% of follicles showing } \\
\text { comedones }\end{array}$} & \multicolumn{2}{|c|}{ Comedone score } \\
\hline & Pre & Post & Pre & Post & Pre & Post & Pre & Post & Pre & Post \\
\hline No treatment & $13.9 \pm 1.7$ & $\mid 3.4 \pm 1.3$ & $0.93 \pm 0.29$ & $1.00 \pm 0.32$ & 20.8 & 20.4 & $6.14 \pm 1.68$ & $6.90 \pm 2.12$ & $0.25 \pm 0.07$ & $0.28 \pm 0.09$ \\
\hline Acetulan & $12.4 \pm 1.6$ & $11.8 \pm 1.3$ & $0.93 \pm 0.06$ & $1.17 \pm 0.31 *$ & 19.9 & $12.7^{*}$ & $6.21 \pm 1.49$ & $8.30 \pm \mid .4 I^{*}$ & $0.25 \pm 0.06$ & $0.33 \pm 0.06^{*}$ \\
\hline Rescue Gel & $13.2 \pm 1.6$ & $13.0 \pm 1.4$ & $0.77 \pm 0.10$ & $0.83 \pm 0.12$ & 17.5 & 16.2 & $5.6 \mathrm{I} \pm 0.45$ & $5.97 \pm 0.42$ & $0.22 \pm 0.02$ & $0.24 \pm 0.02$ \\
\hline
\end{tabular}

Notes: The comedone score was determined by follicular biopsies both pretreatment and posttreatment following thrice weekly applications of either no treatment (negative control), Acetulan (positive control), or Rescue Gel for a total of 6 weeks to the backs of volunteers. $* P<0.05$ versus no treatment. Data are presented as mean \pm SEM.

Abbreviation: Rescue Gel, QV Face Rescue Gel; SEM, standard error of the mean. 


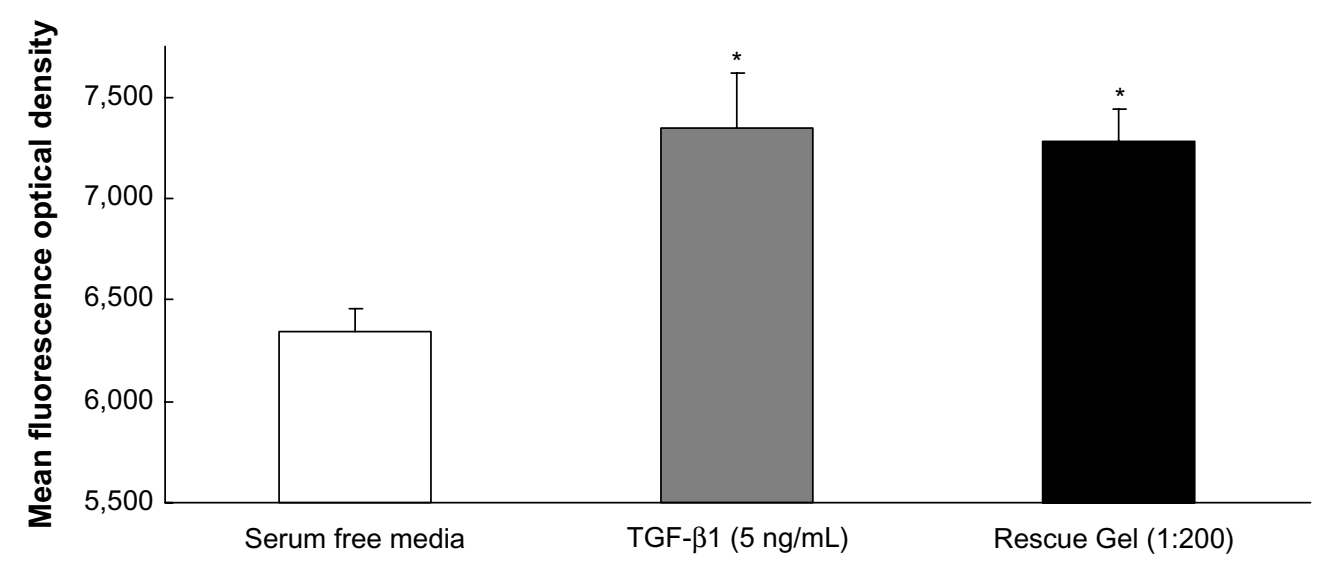

Figure I Mean fluorescence optical density measured in cultured neonatal human foreskin fibroblasts treated with TGF- $\beta$ I (positive control, $n=8$ ) or with or without Rescue Gel (I:200) following immunofluorescence staining for collagen type I.

Note: $* P<0.001$ versus serum free media.

Abbreviations: TGF- $\beta$ I, transforming growth factor beta I; Rescue Gel, QV Face Rescue Gel.

increase in comedogenic scores for the positive control test sites treated with Acetulan compared with no treatment $(P<0.0001)$. The results of sites treated with Rescue Gel exhibited a difference of $7.5 \%$ in comedogenic activity relative to pretreatment baseline. When compared to the untreated negative control site, which showed a change in the comedogenic score of $2.1 \%$, and the positive control Acetulan, which showed a change of $55.8 \%$, Rescue Gel exhibited scores that are considered to be noncomedogenic.

\section{Discussion}

In this study, Rescue Gel was shown to significantly induce expression of collagen types I and III in cultured HFFs at a dilution of 1:200 similarly to TGF- $\beta 1$, a potent inducer of collagen types $\mathrm{I}^{6}$ and III. ${ }^{7}$ Collagen is a major structural protein in skin. It gives skin its strength and durability and is responsible for the smooth, plump appearance of young, healthy skin, while being degraded and reduced in aged skin. ${ }^{5}$ Aged skin is more susceptible to infection, reduced wound repair capacity, and has an increased susceptibility to tear injuries. ${ }^{11}$

This increase in collagen expression is likely due to the inclusion of niacinamide in Rescue Gel which has been shown to stimulate collagen and ceramide synthesis and keratinocyte differentiation, all leading to improved skin appearance and epidermal barrier function. Topical niacinamide has also been shown to have anti-inflammatory properties. ${ }^{12}$ Further, the topical application of niacinamide to sensitive skin with an abnormal skin barrier function has been shown to improve TEWL, reduce stinging, increase skin hydration, and reduce abnormal desquamation, possibly by an increase in intercellular lipid synthesis in the stratum corneum. ${ }^{13}$

Rescue Gel was found to reduce erythema induced by solar-simulated UV on the skin similarly to $0.5 \%$ hydrocortisone (positive control). Anti-inflammatory ingredients

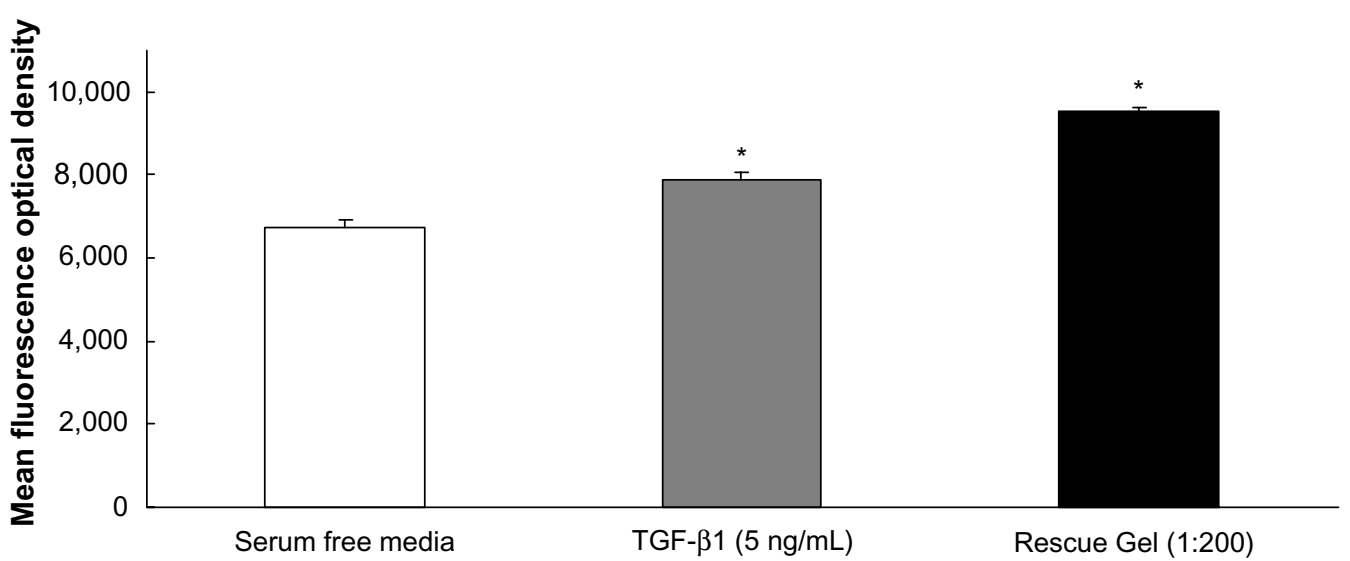

Figure 2 Mean fluorescence optical density measured in cultured neonatal human foreskin fibroblasts treated with TGF- $\beta$ I (positive control, $n=8$ ) or with or without Rescue Gel (I:200) following immunofluorescence staining for collagen type III.

Note: $* P<0.0001$ versus serum free media.

Abbreviations: TGF- $\beta$ I, transforming growth factor beta I; Rescue Gel, QV Face Rescue Gel. 


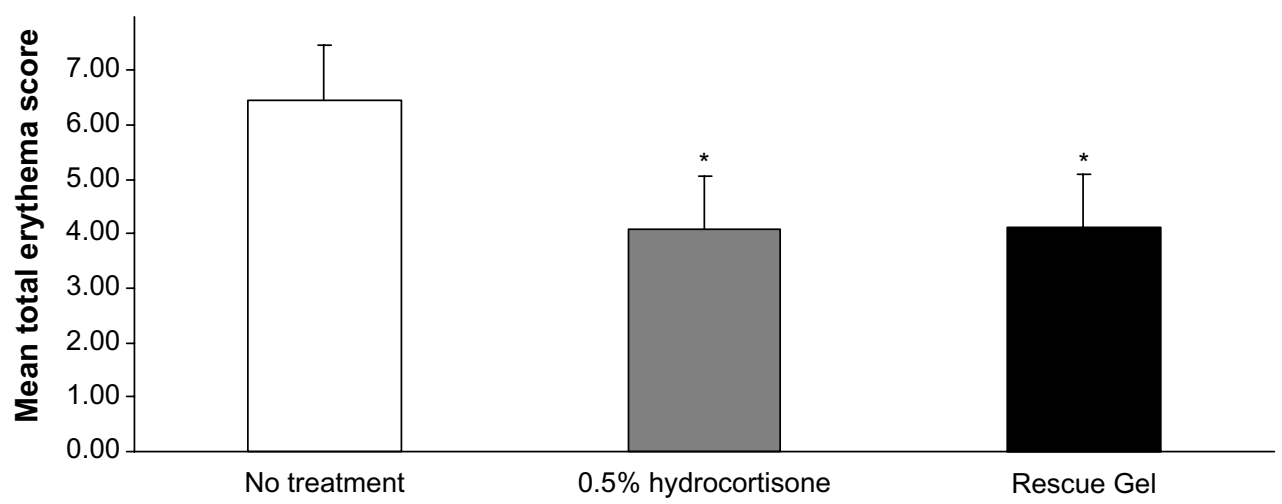

Figure 3 Mean total erythema scores evaluated and scored by visual comparison of subsites on the backs of volunteers.

Notes: Volunteers received either no treatment, $0.5 \%$ hydrocortisone cream (positive control), or Rescue Gel applied to the skin at a dose of $4 \mathrm{mg} / \mathrm{cm}^{2}$ immediately following a series of UV-simulated irradiations with I-second increments (three below the MED, one at the MED, and one above the MED), and again 4-5 hours later. $* P<0.05$ versus no treatment.

Abbreviations: UV, ultraviolet; MED, minimal erythemal dose; Rescue Gel, QV Face Rescue Gel.

are important in formulations for the treatment of sensitive skin to reduce redness and stinging, which will also increase consumer tolerance. ${ }^{4}$

Rescue Gel contains niacinamide, $H$. virginiana (witch hazel) water, DLTP, and A. sativa (oat) kernel extract as antiinflammatory ingredients. $H$. virginiana has astringent properties that help to cleanse the skin and provide a cooling effect. ${ }^{14}$ Studies have shown $H$. virginiana to be beneficial in reducing UV-induced erythema, ${ }^{15,16}$ as well as reducing itching, erythema, and scaling in patients with moderate-to-severe atopic eczema. ${ }^{17}$ DLTP (a form of Vitamin E) has been shown to be effective in preventing and improving redness from UV exposure, as well as reducing a wide range of inflammatory symptoms. ${ }^{18} A$. sativa (oat) kernel extract is used for the protection and alleviation of rashes and dry skin, and for cleansing and moisturising. ${ }^{19}$ In addition to anti-inflammatory properties, colloidal oatmeal also exhibits antioxidant, antiirritant, and immunomodulatory effects. ${ }^{19}$

Rescue Gel was also shown to reduce TEWL compared to baseline levels. Regular use of moisturizing ingredients improves skin hydration and protects sensitive skin. Skin moisturization benefits itchiness, dryness, and scaling.

Ingredients in Rescue Gel that aid in skin moisturization include glycerin, paraffinum liquidum, and panthenol. Glycerin is a humectant and emollient commonly used to moisturize dry skin. ${ }^{20}$ Glycerin draws transepidermal water to the surface of the skin, maintaining stratum corneum hydration. ${ }^{20}$ Paraffinum liquidum is adsorbed on the skin and provides an emollient film that reduces TEWL from the stratum corneum. ${ }^{21}$ Panthenol promotes lipid synthesis to

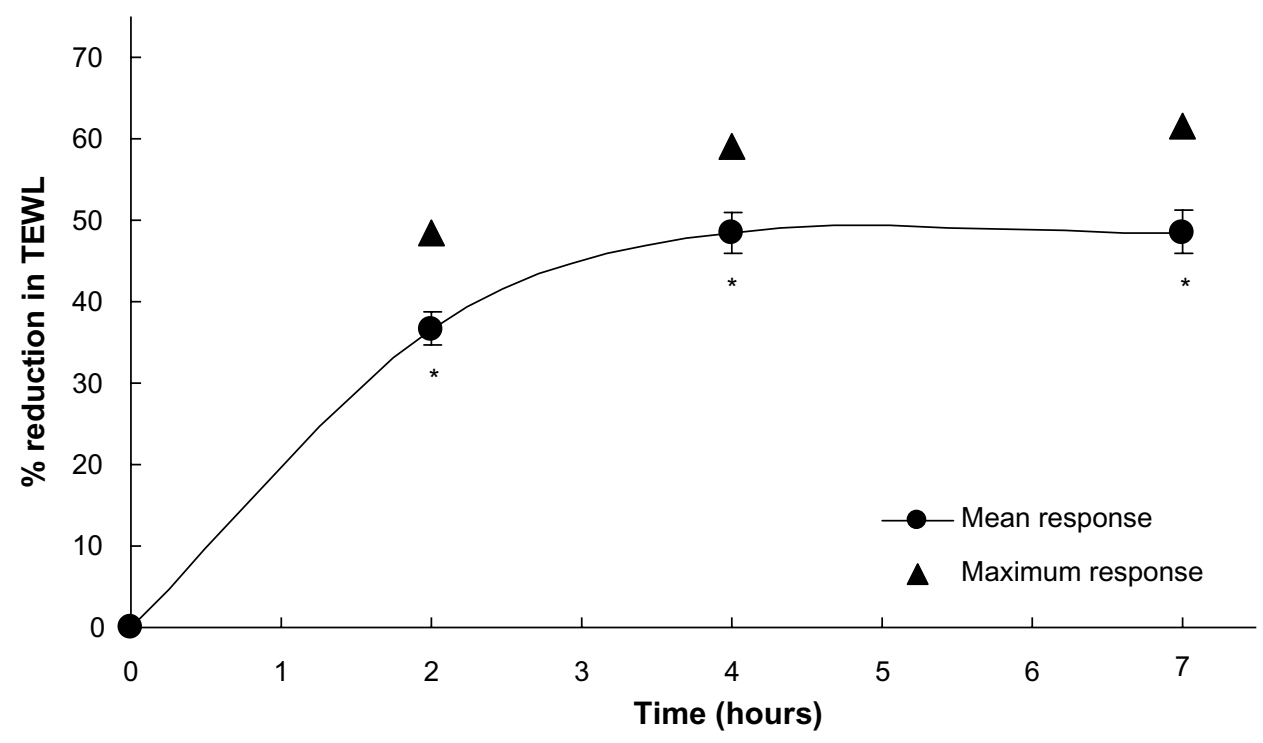

Figure 4 Mean percentage reduction in TEWL measurements for Rescue Gel over time (hours).

Notes: The maximum response at each time point is also shown in the figure. ${ }^{*} P<0.05$ versus $t=0$.

Abbreviations: TEWL, transepidermal water loss; Rescue Gel, QV Face Rescue Gel. 
improve skin barrier function. ${ }^{22}$ Additionally, panthenol is used as a humectant and emollient to improve stratum corneum hydration, reduce TEWL, and to maintain skin softness and elasticity. ${ }^{22}$

It is important that products designed for use on sensitive skin minimize exacerbation of the symptoms associated with this condition. Rescue Gel was also found in this study to be nonstinging, nonirritating, nonsensitizing, and noncomedogenic. Further, to reduce irritation potential, Rescue Gel is also fragrance, lanolin, and propylene glycol free.

During the past several years, sensitive skin has become an important field in dermatology, with a large proportion of the population affected. Over this time, the number of consumer products targeted toward this group has increased substantially; however, there is still a paucity of documented studies in this field. The combination of ingredients specifically selected for the formulation of Rescue Gel has been shown in this study to exhibit attributes that make it effective for use on sensitive skin, including the ability to induce collagen in vitro, reduce erythema, and reduce TEWL, and has been formulated to be nonstinging, nonirritating, nonsensitizing, and noncomedogenic.

\section{Acknowledgement}

This study was supported by the Wound Management Innovation Cooperative Research Centre, West End, Queensland, Australia.

\section{Disclosure}

Ingrid R Heinicke, Tanya M Barnes, and Kerryn A Greive are employed by Ego Pharmaceuticals, the sponsor of the study and manufacturer of QV Face Rescue Gel. The authors report no other conflicts of interest in this work.

\section{References}

1. Misery L, Sibaud V, Merial-Kieny C, Taieb C. Sensitive skin in the American population: prevalence, clinical data, and role of the dermatologist. Int J Dermatol. 2011;50(8):961-967.

2. Inamadar AC, Palit A. Sensitive skin: an overview. Indian J Dermatol Venereol Leprol. 2013;79(1):9-16.
3. Farage MA, Maibach HI. Sensitive skin: closing in on a physiological cause. Contact Dermatol. 2010;62(3):137-149.

4. Draelos ZD. Sensitive skin: perceptions, evaluation, and treatment. Contact Dermat. 1997;8(2):67-78.

5. Naylor EC, Watson RE, Sherratt MJ. Molecular aspects of skin ageing. Maturitas. 2011;69(3):249-256.

6. Cutroneo KR. TGF-beta-induced fibrosis and SMAD signaling: oligo decoys as natural therapeutics for inhibition of tissue fibrosis and scarring. Wound Repair Regen. 2007;15(Suppl 1):S54-S60.

7. Bertelli R, Valenti F, Oleggini R, et al. Cell-specific regulation of alpha1(III) and alpha2(V) collagen by TGF-beta1 in tubulointerstitial cell models. Nephrol Dial Transplant. 1998;13(3):573-579.

8. Ibbotson SH, Diffey BL, Farr PM. The effect of topical indomethacin on ultraviolet-radiation-induced erythema. Br J Dermatol. 1996; 135(4):523-527.

9. Frosch PJ, Kligman AM. A method for appraising the stinging capacity of topically applied substances. J Soc Cosmet Chem. 1977; 28(5):197-209.

10. Mills OH, Kligman AM. A human model for assessing comedogenic substances. Arch Dermatol. 1982;118(11):903-905.

11. Farage MA, Miller KW, Elsner P, Maibach HI. Characteristics of the aging skin. Adv Wound Care. 2013;2(1):5-10.

12. Matts PJ, Oblong JE, Bissett DL. A review of the range of effects of niacinamide in human skin. IFSCC Magazine. 2002;5:285-289.

13. Yakota T, Matsumoto M, Sakamaki T, et al. Classification of sensitive skin and development of a treatment system appropriate for each group. IFSCC Magazine. 2003;6:303-307.

14. Sweetman SC, editor. Martindale. The Complete Drug Reference. 35th ed. London: The Pharmaceutical Press; 2006:2103.

15. Korting HC, Schafer-Korting M, Klovekron W, et al. Comparative efficacy of Hamamelis distillate and hydrocortisone cream in atopic eczema. Eur J Clin Pharmacol. 1995;48(6):461-465.

16. Hughes-Formella BJ, Filbry A, Gassmueller J, Rippke F.Anti-inflammatory efficacy of topical preparations with $10 \%$ Hamamelis distillate in a UV erythema test. Skin Pharmacol App Skin Physiol. 2002;15(2): 125-132.

17. Hughes-Formella BJ, Bohnsack K, Rippke F, et al. Anti-inflammatory effect of Hamamelis lotion in a UVB erythema test. Dermatology. 1998; 196(3):316-322.

18. Rerek M, Botschka E, Gorcea M, et al. Vital ET ${ }^{\mathrm{TM}}$ : a potent new ingredient for effective delivery of tocopherol phosphates. Cosmet Sci Technol. 2004;75-83.

19. Wu J. Anti-inflammatory ingredients. J Drugs Dermatol. 2008; 7(7 Suppl):S13-S16.

20. Bissett DL, McBride JF. Skin conditioning with glycerol. J Soc Cosmet Chem. 1984;35(7):345-350.

21. Taylor EA. Cutaneous adsorption of bath oils. Arch Dermatol. 1963;87: 369-371.

22. Ebner F, Heller A, Rippke F, Tausch I. Topical use of dexpanthenol in skin disorders. Am J Clin Dermatol. 2002;3(6):427-433.
Clinical, Cosmetic and Investigational Dermatology

\section{Publish your work in this journal}

Clinical, Cosmetic and Investigational Dermatology is an international, peer-reviewed, open access, online journal that focuses on the latest clinical and experimental research in all aspects of skin disease and cosmetic interventions. All areas of dermatology will be covered; contributions will be welcomed from all clinicians and

\section{Dovepress}

basic science researchers globally. This journal is indexed on CAS The manuscript management system is completely online and includes a very quick and fair peer-review system, which is all easy to use. Visit http://www.dovepress.com/testimonials.php to read real quotes from published authors. 